\title{
Eulerian model for the prediction of nucleate boiling of refrigerant in heat exchangers
}

\author{
D. Simón, M. C. Paz, A. Eirís \& E. Suárez \\ E.T.S. Ingenieros Industriales, University of Vigo, Spain
}

\begin{abstract}
In this article, a boiling model for "Fluent 6" fluid flow simulation software, for its use in gas-liquid heat exchangers, is presented. After a thorough study of existing bibliographies, a physical model has been compiled, which has been incorporated into the eulerian multiphase model, the most suitable of those available in Fluent for the resolution of these kinds of multiphase flows. Also discussed are some of the aspects of the implementation, which basically consist of the modeling of the interactions between phases as source terms in the governing equations solved by Fluent. Finally, the implementation of the model has been validated against experimental tests from the available literature, achieving a satisfactory degree of concordance.
\end{abstract}

Keywords: CFD, heat exchangers, nucleate boiling, FLUENT, multiphase, eulerian.

\section{Introduction}

The study of boiling is one of the requirements in the design stages. The experimental tests in this field are complex and very costly, which means that a computer tool is necessary to make quick and economical predictions.

The boiling model presented in this paper is physically valid for the region of nucleate or sub-cooled boiling. The equations shown are based on local parameters in order to be applied to any geometry, having undertaken the necessary generalizations from the correlations, which were originally obtained in experimental tests. A modification of the Kurul and Podowski [1] model is employed, which has successfully been applied to diverse geometries by several authors. 
The selected multiphase model of those available in FLUENT is the eulerian, which fully resolves all the momentum and energy equation for every fluid in the simulation. Nevertheless, the treatment of the turbulence is independent from the general multiphase model, and the $\kappa$-epsilon mixture model has been chosen here, which solves the turbulent variables for a mixture fluid.

The presentation of the model is divided into two parts. Firstly, the interactions between gasses and liquids in bubbly flows, and secondly, the interactions derived from phase changes.

\section{Interactions between gasses and liquids in bubbly flows}

\subsection{Model}

This part of the model is comprised mainly of all the interaction forces that are needed to properly describe the mean flow of gas bubbles in a liquid flow. The force between phases is decomposed as a sum of the following terms (all of the following terms are forces over the gas phase per unit of mixture volume):

\section{Drag force}

$$
\overrightarrow{F_{D}}=\frac{3}{4} \frac{C_{D}}{d_{b}} \alpha_{g} \rho_{l}\left(\vec{u}_{l}-\vec{u}_{g}\right)
$$

The expression for the drag coefficient is taken from Ishii and Chawla [2]:

$$
C_{D}=\max \left(\frac{24\left(1+0.1 \cdot R e_{b}^{0.75}\right)}{R e_{b}} \quad, \quad \frac{4}{3} d_{b} \sqrt{\frac{\left(\rho_{l}-\rho_{g}\right) g}{\left(1-\alpha_{g}\right) \sigma_{l}}}\right)
$$

Force of gravitational origin Given that the difference of densities between the liquid and vapour phases is very high, the body forces of a gravitational type cause significant effects on the paths of the bubbles and in the dead areas which they form.

\section{Lift force}

$$
\overrightarrow{F_{L}}=C_{L} \alpha_{g} \rho_{l}\left(\overrightarrow{u_{g}}-\overrightarrow{u_{l}}\right) \times\left(\vec{\nabla} \times \overrightarrow{u_{l}}\right)
$$

Lift coefficient The value of the lift coefficient is one of the most controversial aspects of this model. In this study, a constant value of 0.25 have proved to be the most adequate which is close to the value of 0.288 that would be obtained using the model presented in [3, 4]. When it is increased over a certain value, particular for each case, its effect on the distribution of the void fraction stops getting stronger, meanwhile the convergence of the problem is negatively affected.

Wall lubrication force It models the force of repulsion which pushes the bubbles when they travel near a wall, due to the areas of high pressure which appear between both. 
This force has been correlated only for adiabatic flows, where it is a necessary interaction as it reproduces the peak in void fractions near the walls. However, it is still in discussion for the modelling of boiling phenomena, because peaks in vapour fractions are not usually observed at high pressures or with some refrigerants [5, 6].

The model to be used here is the Antal et al. [7] model, in the way expressed by Frank et al. [8].

$$
\begin{gathered}
{\overrightarrow{F_{l v}}}^{w}=-\frac{C_{W L}}{d_{b}} \alpha_{v} \rho_{l}\left\|\vec{u}_{r e l}^{*}\right\|^{2} \overrightarrow{n_{w}} \\
C_{W L}=\max \left[0, C_{W 1}+\frac{C_{W 2}}{y_{w}} d_{b}\right] \\
\vec{u}_{r e l}^{*}=\overrightarrow{u_{r e l}}-\left(\overrightarrow{u_{r e l}} \cdot \overrightarrow{n_{p}}\right) \overrightarrow{n_{p}}
\end{gathered}
$$

The parameter $C_{W L}$ makes this force inversely proportional to the distance from the wall. $C_{W 1}$ and $C_{W 2}$ can have different values according to the author consulted and the experiment that is being simulated. Here, two sets of parameters are tested: on one hand $C_{W 1}=-0.02$ and $C_{W 2}=0.04$, and on the other hand $C_{W 1}=-0.01$ and $C_{W 2}=0.05$, which have shown to give the best concordance.

Turbulence model The presence of bubbles in the flow modifies the turbulence from the monophase values for the liquid flow. This effect is introduced in the model in two ways: increasing the turbulent viscosity of the mixture and adding an extra diffusive force.

$$
\overrightarrow{F_{D}}=-C_{T D} \rho_{l} \kappa_{l} \vec{\nabla} \alpha_{v}
$$

where $C_{T D}$ is the turbulent dispersion coefficient, which takes the value of 0.1 in accordance with Kurul and Podowski [1].

$$
\mu_{l}^{e f}=\mu_{l}+\mu_{l}^{t u r b}+\mu_{l}^{b u b}
$$

$\mu_{l}^{b u b}$ is the bubble-induced turbulent viscosity. Following Sato [9]:

$$
\mu_{l}^{b u b}=C_{\mu b} \rho_{l} \alpha_{v} d_{b}\left\|\overrightarrow{u_{v}}-\overrightarrow{u_{l}}\right\|
$$

\section{Mass and thermal transferences model}

\subsection{Wall model}

It has been considered that the heat which goes through the wall is divided into two types: latent heat associated with the formation of vapour and convective heat to the liquid. The convective heat is increased by the formation of bubbles. When a bubble is detached from the wall, its exit forces its place to be filled by liquid, which comes from areas further away from the wall and, therefore, cooler. In order to take into account this effect, the area of wall-liquid contact has been divided into two fractions of wall with different laws of convection: one is the area where the 
convection heat is influenced by the exit of bubbles $\left(A_{b u b}\right)$, and the other is where the heat flux is not affected by the boiling $\left(1-A_{b u b}\right)$. In this way:

$$
q_{w}=q_{c o n v}+q_{e}=\left(q_{\phi}+q_{Q}\right)+q_{e}
$$

where $q_{w}$ is the total wall heat flux per unit area, $q_{e}$ is the evaporation heat flux and $q_{c o n v}$ is the single-phase convection heat flux, which, in turn comprises two terms: $q_{\phi}$ the convection component outside the influence of nucleating bubbles and $q_{Q}$, the quenching heat flux or the pumping heat flux, the part of the heat flux which is affected by the boiling.

$$
\begin{gathered}
q_{\phi}=h_{\phi}\left(1-A_{b u b}\right)\left(T_{w}-T_{l, 1^{\circ}}\right) \\
q_{Q}=h_{Q}\left(A_{b u b}\right)\left(T_{w}-T_{l, \text { free }}\right) \\
q_{e}=\dot{m}_{w}\left(h_{l g}+c_{p l}\left(T_{s}-T_{l}\right)\right)
\end{gathered}
$$

The bubble influence area per unit wall area is given by:

$$
A_{b u b}=\min \left[1, N_{a} K\left(\frac{\pi d_{b w}^{2}}{4}\right)\right]
$$

$N_{a}$ is the density of active nucleation sites. The correlation used has been created by Lemmert and Chawla [6].

$K$ is a factor of influence whereby the area of each bubble projected on the wall should be multiplied in order to obtain the area of influence around each bubble in which the quenching heat transfer acts. Usually $K=4$ [10] is taken. The maximum of $A_{b u b}$ has been limited to 1.0.

The quenching heat transfer coefficient is given by:

$$
h_{Q}=\frac{2}{\sqrt{\pi}} f \sqrt{t_{\text {wait }} k_{l} \rho_{l} c_{p l}}
$$

$f \quad$ is the nucleation frequency, whose expression has been proposed by Cole [11]:

$$
f=\sqrt{\frac{4 g\left(\rho_{l}-\rho_{v}\right)}{3 d_{b p} \rho_{l}}}
$$

$t_{\text {wait }}$ is the waiting time [1]. It is the time which elapses between when a bubble detaches from the wall and another starts to grow in the same nucleation site

$T_{\text {free,l }}$ is the local free stream temperature. It is the value of the temperature of the liquid out of the thermal layer, assigned to a point of the wall. In order to obtain this value, an algorithm capable to climb prismatic cell layers have been developed. In this way, if a prismatic boundary layer is attached to the mesh, the program will be able to climb the boundary layer until smooth thermal gradients are reached.

The bubble departure diameter, $d_{b w}$, is modeled according to the correlation of Unal [12] in the way expressed by Yeoh and Tu [13]. 
Vapour mass generation rate per unit area of heated wall This is calculated in a mechanistic way, as a product of the mass of a bubble by the number of them which leave the wall per unit of time and area of wall. Its sign will be positive, in the direction of the formation of vapour.

$$
\dot{m}_{w}=\left(\frac{\pi d_{b w}^{3}}{6}\right) \rho_{v} f N_{a}
$$

\subsection{Model in the bulk bubbly flow}

The vapour phase is supposed to always be at its saturation temperature, $T_{v}=T_{s}$. In this way, the heat exchange between vapour and liquid can be expressed as:

$$
q_{l g}=\min \left(I H T^{\prime} A_{i}\left(T_{l}-T_{s}\right), 0\right)
$$

so the mass transfer will be:

$$
\begin{gathered}
\dot{m}_{l v}=\frac{q_{l v}}{h_{l g}} \\
I H T^{\prime}=\frac{k_{l}}{d_{b}} N u_{b}
\end{gathered}
$$

The Nusselt number for the bubbles $N u_{b}$ is obtained from the correlation of Ranz and Marshall [14]:

$$
N u_{b}=2+0.6 \operatorname{Re}_{b}^{1 / 2} \operatorname{Pr}_{l}^{1 / 3}
$$

The diameter of the bubbles is modeled with a correlation proposed by Zeitoun and Shoukri [15] for low pressures:

$$
\frac{d_{b}}{\sqrt{\frac{\sigma}{g\left(\rho_{l}-\rho_{g}\right)}}}=\frac{0.0683\left(\rho_{l} / \rho_{g}\right)^{1.326}}{R e^{0.324}\left(J a+\frac{149.2\left(\rho_{l} / \rho_{g}\right)^{1.326}}{B o^{0.487} R e^{1.6}}\right)}
$$

\subsection{Validation}

The code has been validated against the experiment performed by Lee et al. [16]. It consists of an annular tube with an upward flow. Its inner diameter is $19.0 \mathrm{~mm}$ and the outer is $37.5 \mathrm{~mm}$. The total length of the tube is $2375 \mathrm{~mm}$, and it is divided into three parts: a middle part of $1670 \mathrm{~mm}$ with a uniformly heated inner tube, where boiling is meant to happen, and two adiabatic parts that are placed to allow full development of the flow. The vapour volume fraction, liquid and vapour velocities and the diameter of the bubbles are measured in a transverse section placed at $1610 \mathrm{~mm}$ downflow from the start of the middle section. The working fluid is de-mineralized water and its properties have been obtained from the database REFPROP7. The properties (especially the saturation temperature and density of the saturated vapour) are allowed to change with the pressure, which origin is placed at the test section and varies hydrostatically. Three cases with 

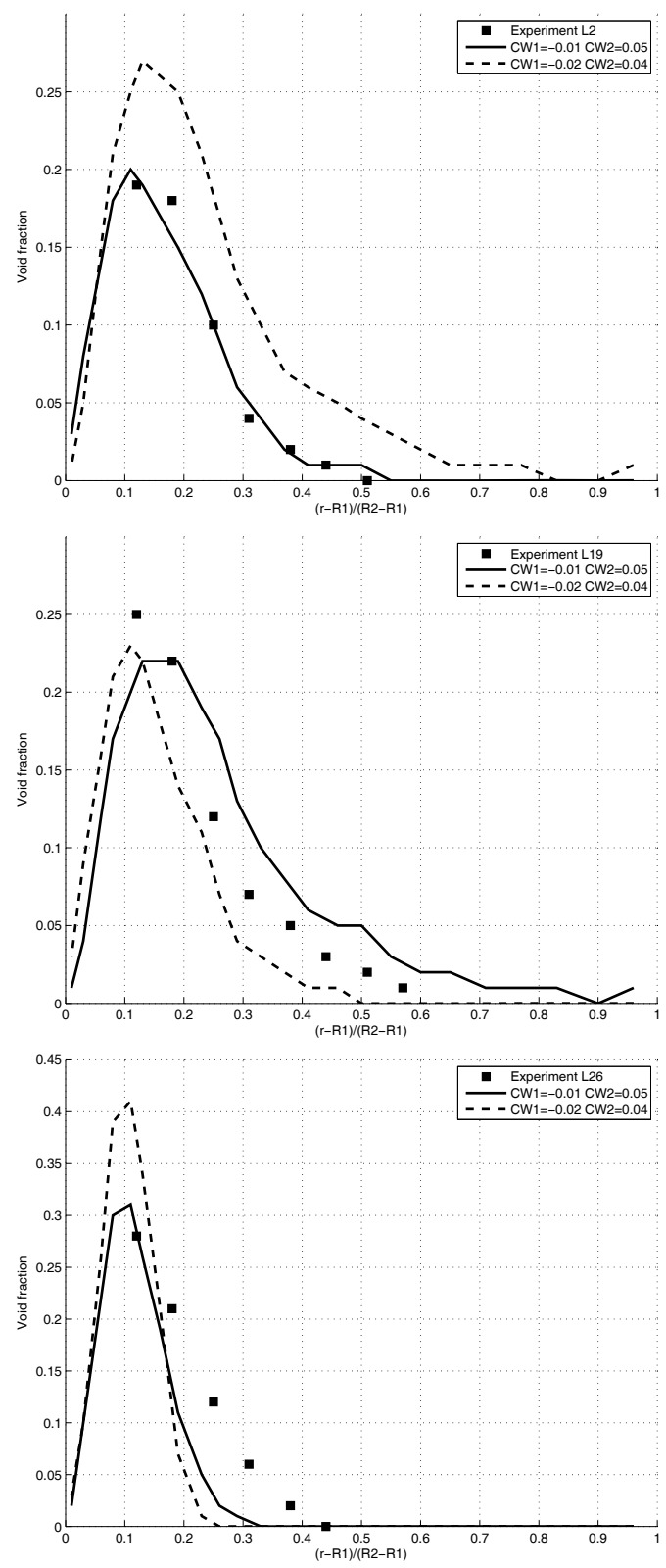

Figure 1: Radial void fraction distribution at the measurement section for all the Lee cases. 

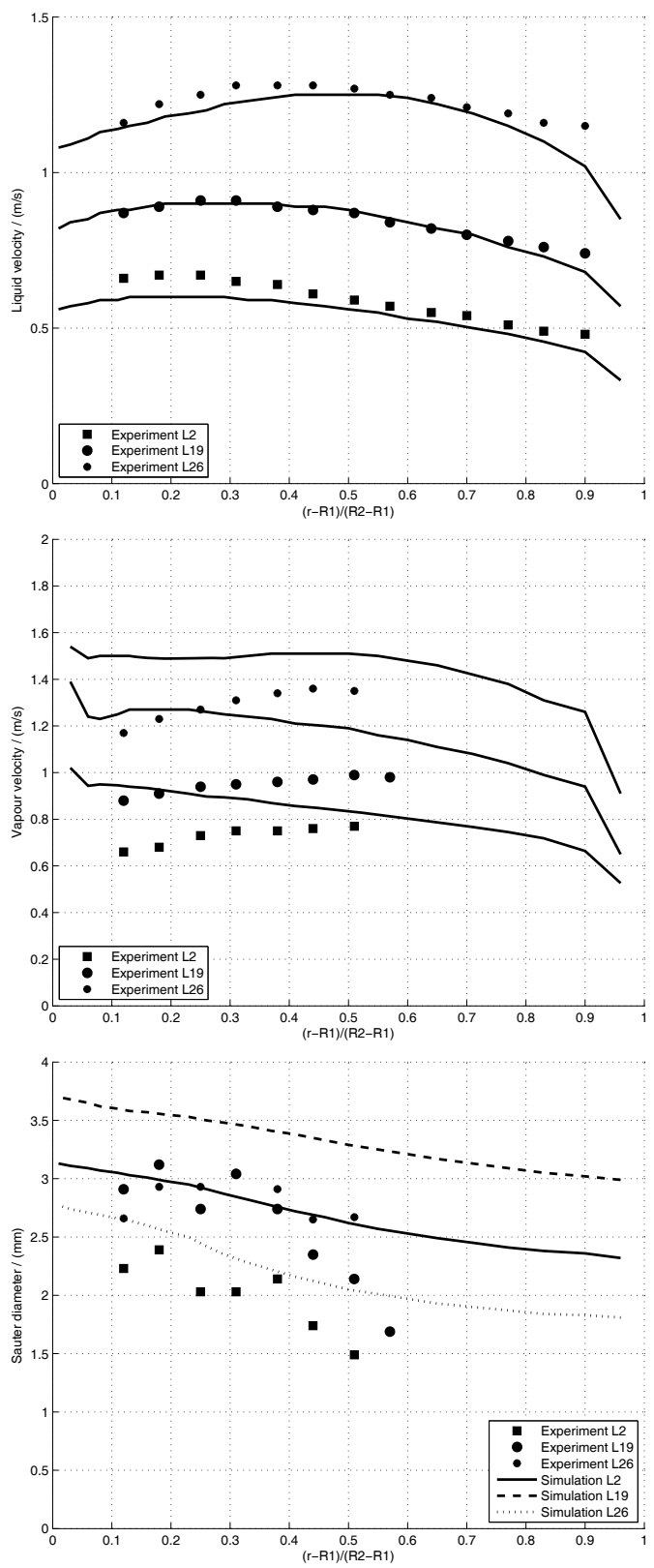

Figure 2: Radial liquid velocity, vapour velocity and bubble diameter distributions at the measurement section for all the Lee cases. 
different mass flows have been selected. The conditions of each one can be seen in table 1 . The problem is again axisymmetric, so this time the mesh is a rectangle of 24 radial elements and 80 longitudinal elements, being the radial sizes increased while going from the heated wall at the ratio: $l_{\{i+1\}} / l_{i}=1.05$.

Table 1: Selected cases from the Lee experiment to perform the validation of the boiling model.

\begin{tabular}{|c|c|c|c|c|c|}
\hline Set & $q_{w}\left(\mathrm{~kW} / \mathrm{m}^{2}\right)$ & $G\left(\mathrm{~kg} / \mathrm{m}^{2} s\right)$ & $T_{l, i n}\left({ }^{\circ} \mathrm{C}\right)$ & $p_{\text {in }}(\mathrm{kPa})$ & $p_{\text {tests }}(\mathrm{kPa})$ \\
\hline \hline Lee 2 & 169.8 & 478.1 & 83.9 & 114.8 & 99.8 \\
\hline Lee 19 & 139.1 & 715.2 & 93.9 & 124.7 & 109.2 \\
\hline Lee 26 & 251.5 & 1059.2 & 90.1 & 134.5 & 117.9 \\
\hline
\end{tabular}

As can be seen, a good level of concordance is obtained with the values: $C_{W 1}=-0.01, C_{W 2}=0.05, C_{L}=0.25$.

In order to achieve convergence the following strategy has been adopted: the evaporation is under relaxed several orders of magnitude and it is limited in first iterations, growing slowly from zero to its final value. The continuity equations (void fraction) and condensation must be greatly relaxed too, but always one order of magnitude less relaxed than the evaporation. If the condensation is not allowed to have a quicker response than the evaporation, too big imbalances between them will appear, which may eventually cause the divergence of the problem.

\section{Conclusions}

The above presented model has proved to be adequate to simulate flow nucleate boiling phenomena, but at the cost of requiring a high computational cost. Some leaks are evident: the diameter of the bubbles needs to be modeled with a transport equation, instead of using a correlation only valid for tubes; the flow variables increase highly their coupling, so the convergence of the problem is negatively affected; and the values of some constants are still in discussion. Anyway, it is the unique model accurate enough without raising the computational cost to prohibitive levels.

\section{References}

[1] Kurul, N. \& Podowski, N.Z., Multidimensional effects in forced convection subcooled boiling. Proceedings of the Ninth International Heat Transfer Conference, August 19-24, Jerusalem, Israel, Vol. 2, pp. 21-26, 1990.

[2] Ishii, M. \& Chawla, T.C., Local drag laws in dispersed two-phase flow. Argonne Repor Anl, 79, pp. 105,52, 1979. 
[3] Tomiyama, A., Tarnai, H., Zun, I. \& Hosokama, S., Transverse migration of single bubbles in simple shear flow. Chem Eng Sci, Vol.57, pp. 1849-1858, 2002.

[4] Ohnuki, A. \& Akimoto, H., Model development for bubble turbulent diffusion and bubble diameter in large vertical pipes. $J$ Nuclear Science Technology, Vol.38, No.12, pp. 1074-1080, 2001.

[5] Krepper, E., Koncar, B. \& Egorov, Y., Cfd modelling of subcooled boiling - concept, validation and application to fuel assembly design. Nuclear Engineering and design, 237, pp. 716-731, 2007.

[6] Lemmert, M. \& Chwala, J., Influence of flow velocity on surface boiling heat transfer coefficient. Heat Transfer in Boiling, Academic Press and Hemisphere, 1977.

[7] Antal, S.P., Lahey, R.T. \& Flaherty, J.E., Analysis of phase distribution in fully developed laminar bubbly two-phase flow. International Journal of Multiphase Flow, 7, pp. 635-652, 1991.

[8] Frank, T., Prasser, H.M., Beyer, M. \& Issa, S.A., Gas-liquid flow around an obstacle in a vertical pipe, cfd simulation \& comparison to experimental data. 6 International Conference on Multiphase Flow, ICMF 2007, Leipzig, Germany, 2007.

[9] Sato, Y., Sadatomi, M. \& Sekoguchi, K., Momentum and heat transfer in two-phase bubble flow - i. theory. Int J Multiphase Flow, 7, pp. 167-177, 1981.

[10] Han, C.Y. \& P.Griffith, The mechanisms of heat transfer in nucleate boiling the heat flux temperature difference relationship. Int J Heat Mass Transfer, 8, pp. 905-914, 1965.

[11] Cole, R., Photographic study of boiling in the region of critical heat flux. AIChE J, 6, pp. 533-542, 1960.

[12] Unal, H., Maximum bubble diameter, maximum bubble-growth time and bubble-growth rate. Int J Heat Mass Transfer, 19, pp. 643-649, 1976.

[13] Yeoh, G.H. \& Tu, J.Y., Two-fluid and population balance models for subcooled boiling flow. Applied Mathematical Modelling, 30, pp. 13701391, 2006.

[14] Ranz, W.E. \& W. R. Marshall, J., Evaporation from drops, part i. Chem Eng Prog, 48(3), pp. 141-146, 1952.

[15] Zeitoun, O. \& Shoukri, M., Bubble behaviour and mean diameter in subcooled flow boiling. ASME J Heat Transfer, 118, pp. 110-116, 1996.

[16] Lee, T., Yun, B., Park, G., Kim, S. \& Hibiki, T., Local interfacial structure of subcooled boiling flow in a heated annulus. Journal of Nuclear Science and Technology, Vol. 45, No 7, pp. 683-697, 2008. 